\title{
DNA Translocation through Hydrophilic Nanopore in Hexagonal Boron Nitride
}

SUBJECT AREAS:

NANOSCALE BIOPHYSICS

NANOPORES

Received

10 July 2013

Accepted

5 November 2013

Published

21 November 2013

Correspondence and requests for materials should be addressed to X.Y.S. (shanxinyan@ iphy.ac.cn) or X.H.L.

(xhlu@iphy.ac.cn)

\author{
Zhi Zhou, Ying Hu, Hao Wang, Zhi Xu, Wenlong Wang, Xuedong Bai, Xinyan Shan \& Xinghua Lu
}

Beijing National Laboratory for Condensed-Matter Physics and Institute of Physics, Chinese Academy of Sciences, Beijing 100190, People's Republic of China.

Ultra-thin solid-state nanopore with good wetting property is strongly desired to achieve high spatial resolution for DNA sequencing applications. Atomic thick hexagonal boron nitride (h-BN) layer provides a promising two-dimensional material for fabricating solid-state nanopores. Due to its good oxidation resistance, the hydrophilicity of h-BN nanopore device can be significantly improved by UV-Ozone treatment. The contact angle of a KCl-TE droplet on h-BN layer can be reduced from $57^{\circ}$ to $26^{\circ}$ after the treatment. Abundant DNA translocation events have been observed in such devices, and strong DNA-nanopore interaction has been revealed in pores smaller than $10 \mathrm{~nm}$ in diameter. The $1 / f$ noise level is closely related to the area of suspended h-BN layer, and it is significantly reduced in smaller supporting window. The demonstrated performance in $\mathrm{h}-\mathrm{BN}$ nanopore paves the way towards base discrimination in a single DNA molecule.

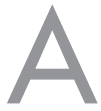

s one of the most promising concepts, DNA sequencing with nanopore has attracted a lot of attention since $1996^{1}$. DNA translocation ${ }^{1-3}$ and base discrimination ${ }^{4}$ has been demonstrated in biological nanopores made of specific proteins. Solid-state nanopores, however, are strongly preferred for higher stability and easier device integration. The first solid-state nanopores are fabricated in silicon nitride $\left(\mathrm{Si}_{3} \mathrm{~N}_{4}\right)$ membranes that are tens of nanometers thick ${ }^{5}$, and significant progress has been made in detection of DNA translocation ${ }^{6,7}$, and DNA motion control ${ }^{8}$ in such nanopore devices. To achieve base discrimination in a single DNA molecule, the effective thickness of a solid-state nanopore has to be reduced down to sub-nanometer. For such propose, atomic thick graphene layers has been employed in fabricating nanopore devices featured with high resolution and geometrical sensitivity ${ }^{9-12}$. On the other hand, the hydrophilicity of nanopore is practically important to avoid clogging due to strong nonspecific hydrophobic interaction with DNA. Recently, the hydrophilicity of graphene nanopores has been improved by atomic-layer deposition of titanium dioxide ${ }^{11}$ and self-assembled hydrophilic coating $^{13}$, which however increases the effective thickness of the pores. New types of atomic thick nanopores with high hydrophilicity are thus still strongly desired.

Hexagonal boron nitride (h-BN) layer, an atomic layered material similar to graphene ${ }^{14}$, is chemically stable with oxidation resistance ${ }^{15}$. In addition, it possesses merits of good thermal conductivity ${ }^{16}$, low dielectric loss and low conductivity (wide band gap energy of $5.97 \mathrm{eV})^{17}$. The single- and few-layer h-BN membranes have been obtained by cleavage $^{18}$, epitaxial growth ${ }^{19,20}$, or chemical synthesis ${ }^{21}$, which provides a new material for nanopore applications. Recently, S. Liu et al. firstly reported the fabrication of solid-state nanopores based on few-layer BN membrane ${ }^{22}$, and totally about one hundred DNA translocation events have been observed. One of the reasons for the limited number of translocation events observed in $\mathrm{BN}$ nanopores is suggested to be the relatively low wetting ability in such material. Here, we show that the hydrophilicity of h-BN nanopores can actually be improved by UV-ozone (UVO) treatment and hundreds of DNA translocation events have been observed in single experimental runs. Our finding thus suggests further promise to utilize h-BN nanopore as ultra-thin solid-state device for future applications.

\section{Results}

Nanopore fabrication in h-BN layers. As sketched in Fig. 1, the single-layer h-BN membranes in our work were grown on copper foils ( $25 \mu \mathrm{m}$ thickness, $99.99 \%$, Alfa Aesar) by decomposition of borane ammonia $\left(\mathrm{BH}_{3}-\mathrm{NH}_{3}\right.$, from Sigma Aldrich) under $1050^{\circ} \mathrm{C}^{19}$. Then, about $300 \mathrm{~nm}$ poly(methyl methacrylate) (PMMA) were coated on the h-BN membranes. After etching off the copper substrate in $\mathrm{FeCl}_{3}$ solution, floating h-BN/PMMA flacks (larger than $1 \mathrm{~mm} \times 1 \mathrm{~mm}$ ) were obtained. The floating flakes were carefully transferred onto a $50 \mathrm{~nm}$ thick $\mathrm{Si}_{3} \mathrm{~N}_{4}$ window, which was prepared by $\mathrm{KOH}$ etching of $\mathrm{Si}_{3} \mathrm{~N}_{4} / \mathrm{Si}$ wafer, as sketched in Fig. $1 \mathrm{~b}$. The size of the $\mathrm{Si}_{3} \mathrm{~N}_{4}$ 


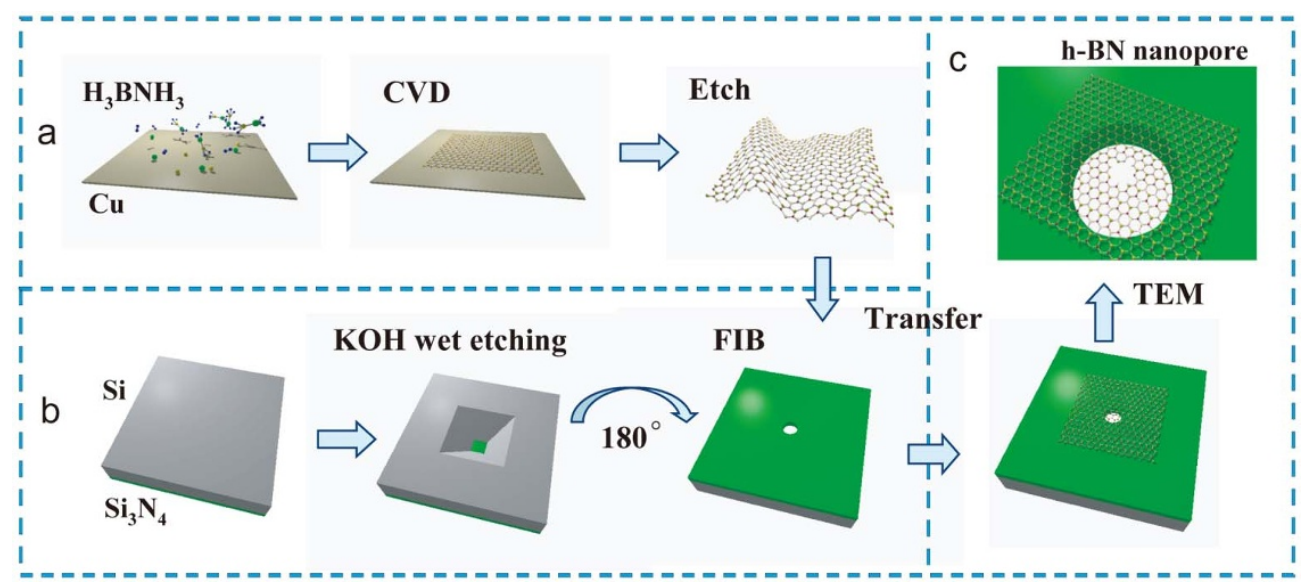

Figure 1 Fabrication process of h-BN nanopore. (a) h-BN membrane was first grown on copper foils by chemical vapor deposition. Then, PMMA was coated on h-BN membrane, and the copper substrate was subsequently etched by $\mathrm{FeCl}_{3}$, leaving h-BN/PMMA floating in solution. (b) Suspended $\mathrm{Si}_{3} \mathrm{~N}_{4}$ window (shown as green) on a pyramidal silicon pit (shown as gray) was formed by anisotropic KOH etching. A $100-1000 \mathrm{~nm}$ sized hole was then drilled on $\mathrm{Si}_{3} \mathrm{~N}_{4}$ window. (c) The floating h-BN/PMMA flake was transferred on to the window, and PMMA was removed by acetone. A nanopore was then fabricated on the suspended h-BN membrane in a TEM.

window is about $20-40 \mu \mathrm{m}$ with a $100-1000 \mathrm{~nm}$ sized hole drilled by focused ion beam (FIB). Nanopore was then drilled on the suspended h-BN membrane with highly focused electron beam in a transmission electron microscope (Model: JEM 2010F).

Characterization of $\mathbf{h}-\mathbf{B N}$ nanopore. Figure 2(a) shows TEM image of a suspended h-BN layer (bright area) on $\mathrm{Si}_{3} \mathrm{~N}_{4}$ window with an $8 \mathrm{~nm}$ nanopore, indicated with a red arrow. Electron energy loss spectra (EELS) were taken on both the suspended h-BN layer (position indicated with blue dashed circle) and the h-BN layer over the $\mathrm{Si}_{3} \mathrm{~N}_{4}$ membrane (red dashed circle), as shown in
Figure 2(b). Clear boron and nitrogen peaks appear in spectrum of suspended h-BN layer (in blue), while strong peaks of silicon and nitrogen are found in spectrum taken over the $\mathrm{Si}_{3} \mathrm{~N}_{4}$ membrane (in red). The carbon peak in the spectrum of suspended h-BN layer is due to the residual amorphous carbon contaminated during layer transferring. Nanopores with diameter from $2 \mathrm{~nm}$ to tens of nanometers are fabricated (see supplementary information, Figure S1) in suspended h-BN layers. Figure 2(c) shows high-resolution TEM image of another nanopore (about $10 \mathrm{~nm}$ in diameter). The FFT of such TEM image shows clear six-fold symmetry (Figure S2), representing the hexagonal structure of the h-BN layer. Raman
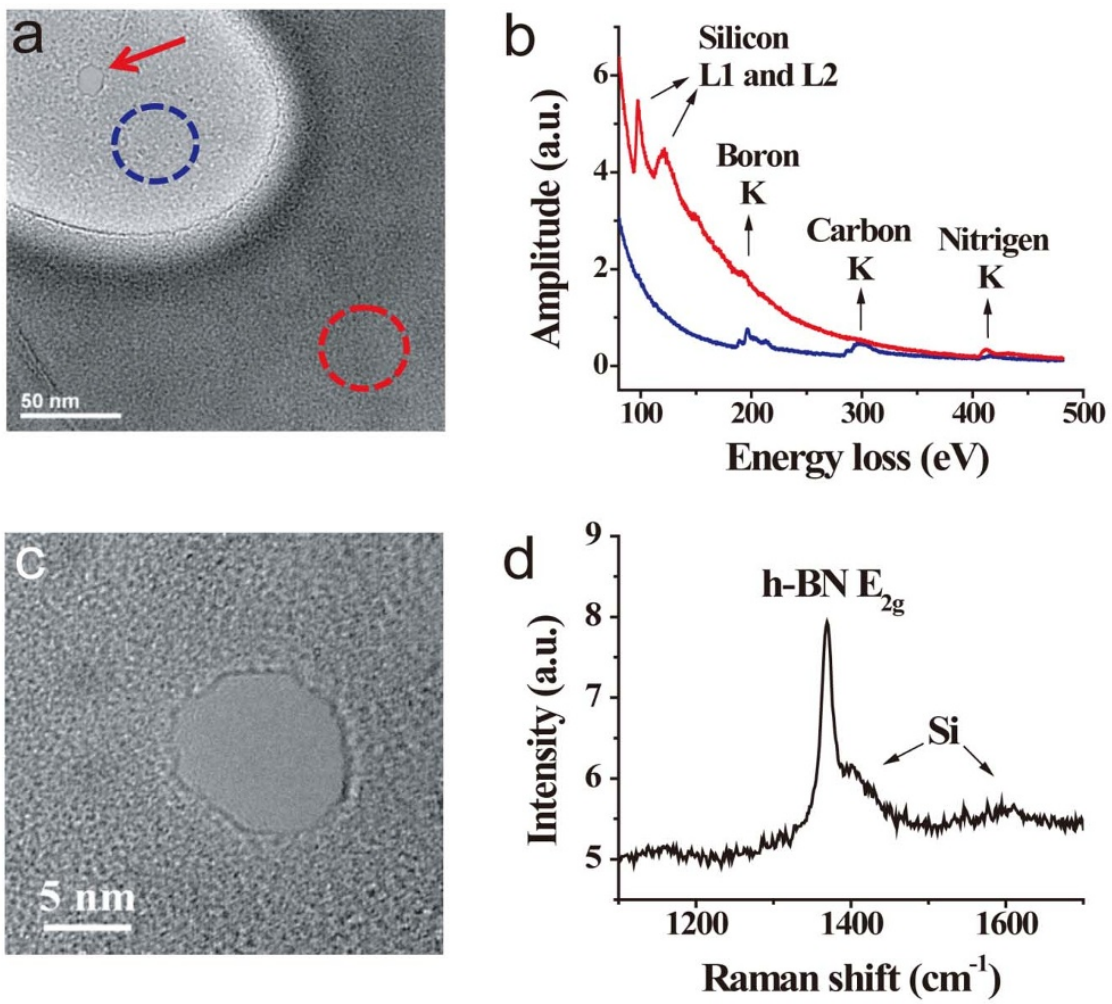

Figure $2 \mid$ (a) TEM image of h-BN membrane (light gray area) on $\mathrm{Si}_{3} \mathrm{~N}_{4}$ substrate (dark gray). The red arrow indicates an 8 nm nanopore. (b) EELS taken in regions of h-BN membrane (position indicated by blue circle in (a)) and $\mathrm{Si}_{3} \mathrm{~N}_{4}$ substrate (red circle). (c) High resolution TEM image of a 10 nm h-BN nanopore. (d) Raman spectrum of h-BN layer transferred onto a Si substrate. 
spectroscopy has been employed to further characterize the h-BN layers. Figure 2(d) shows typical Raman spectrum of h-BN layer transferred onto a silicon wafer (to minimize the background luminescence from $\mathrm{Si}_{3} \mathrm{~N}_{4}$ ), excited with a laser wavelength of $532 \mathrm{~nm}$. It is known that the $\mathrm{E}_{2 \mathrm{~g}}$ peak of a single-layer h-BN is centered at $1369 \pm 1 \mathrm{~cm}^{-1}$, while that for the bi-layer and the bulk are centered at $1365 \pm 2 \mathrm{~cm}^{-1}$ and $1366 \mathrm{~cm}^{-1}$, respectively ${ }^{23}$. The observed Raman peak in our samples is centered at $1369.3 \pm$ $0.1 \mathrm{~cm}^{-1}$ (Figure S3), indicating the presence of single-layer h-BN membrane. The small peaks at $1400 \mathrm{~cm}^{-1}$ and $1600 \mathrm{~cm}^{-1}$ are the third harmonic peaks of the silicon substrate ${ }^{24}$.

Hydrophilicity improvement. The h-BN material is resistant to oxidation and intact after UVO treatment, and such advantage can be used to improve hydrophilicity of h-BN nanopores. We characterized the wetting ability of h-BN layer by measuring the contact angle of a KCl-TE droplet on layer surface and compared it with other materials normally used for fabricating solid-state nanopores. The measurements are taken immediately after 15minute UVO treatment (Jelight Company Inc., model No. 42-220). Figure 3 shows the measured contact angles on $\mathrm{Si}_{3} \mathrm{~N}_{4}$ surface $(50 \mathrm{~nm}$ thick), UVO treated $\mathrm{Si}_{3} \mathrm{~N}_{4}$ surface, h-BN layer on $\mathrm{Si}_{3} \mathrm{~N}_{4}$ substrate, UVO treated h-BN layer on $\mathrm{Si}_{3} \mathrm{~N}_{4}$ substrate, and graphene on $\mathrm{Si}_{3} \mathrm{~N}_{4}$ substrate. The UVO treatment was not applicable to graphene due to its low oxidation resistance ${ }^{25}$. Each measurement was carried out with more than 10 samples. The untreated $\mathrm{h}-\mathrm{BN}$ layer gives a contact angle of $57^{\circ}$, close to that on $\mathrm{Si}_{3} \mathrm{~N}_{4}$ surface $\left(54^{\circ}\right)$. The contact angle on UVO treated h-BN layer decreases to $26^{\circ}$. Such good wetting property can last for at least 30 minutes in ambient lab environment, long enough for device assembling in a typical nanopore experiment. We note that the UVO treated $\mathrm{Si}_{3} \mathrm{~N}_{4}$ membrane shows the lowest contact angle of $6^{\circ}$ and the graphene surface produce a contact angle of $67^{\circ}$, highest among all materials tested in our experiment.

DNA translocation through $\mathbf{h}-\mathrm{BN}$ nanopore. To conduct the DNA translocation experiments, the flow cell, PDMS gaskets and the fabricated h-BN nanopore chips were firstly treated with UVO on both the front and the back side for 15 minutes each and then assembled as fast as possible (typically within 2 minutes). Saline solution ( $1 \mathrm{M} \mathrm{KCl}$, room temperature, $\mathrm{pH} 7.8$ ) was then added to both sides of the chip. A pair of $\mathrm{Ag} / \mathrm{AgCl}$ electrodes supplied a bias voltage $V$ to drive ions through the nanopore and the ionic current was monitored in real-time. Fig. 4(a) is a cartoon picture of such device, and Fig. 4(b) presents a typical I-V curve of a $4 \mathrm{~nm}$ nanopore in saline solution, through which a resistance of $45 \mathrm{M} \Omega$ is derived by

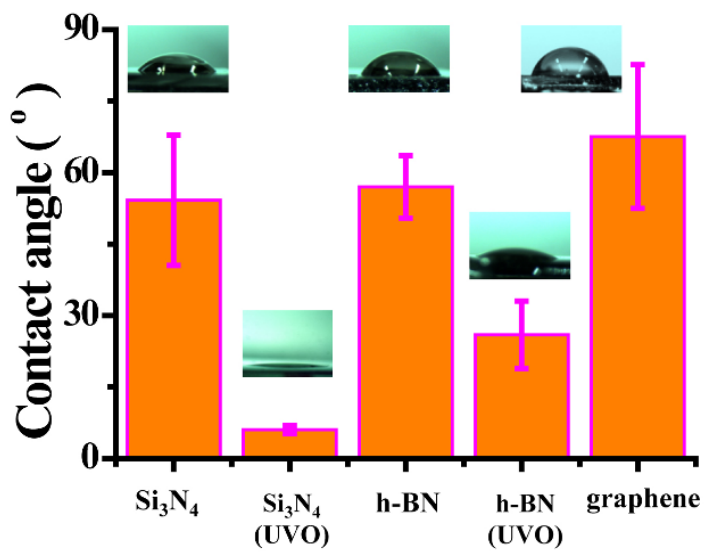

Figure $3 \mid$ Contact angles of droplet ( $1 \mu 11 \mathrm{M} \mathrm{KCl-TE} \mathrm{buffer)} \mathrm{on} \mathrm{surfaces}$ of $\mathrm{Si}_{3} \mathrm{~N}_{4}$ substrate $\left(54^{\circ}\right)$, UVO treated $\mathrm{Si}_{3} \mathrm{~N}_{4}\left(6^{\circ}\right)$, h-BN layer $\left(57^{\circ}\right)$, UVO treated h-BN layer $\left(26^{\circ}\right)$, and Graphene $\left(67^{\circ}\right)$. The insets are typical microscope images. a linear fit. When negative charged double-strand DNA molecules $(\lambda$-DNA from New England Biolabs) were added to one side of the chip, a series of spikes were observed in conductance traces (Fig. 4c), arisen from single DNA molecule translocation through the nanopore. The driving voltage is $100-250 \mathrm{mV}$ and a $30 \mathrm{kHz} 4$ pole Bessel filter was employed during data acquisition and the sampling rate was $250 \mathrm{kHz}$.

Typically, at least hundreds of DNA translocation events can be observed in each nanopore device. Fig. 4(d) shows the scatter plot of conductance blockade $\Delta \mathrm{G}$ versus time duration $\Delta \mathrm{t}$ of each DNA translocation event under driving voltage of $150 \mathrm{mV}$. The right panel is the histogram of conductance blockade, which can be fitted by a single Gaussian peak (blue line). The fitted curve is centered at
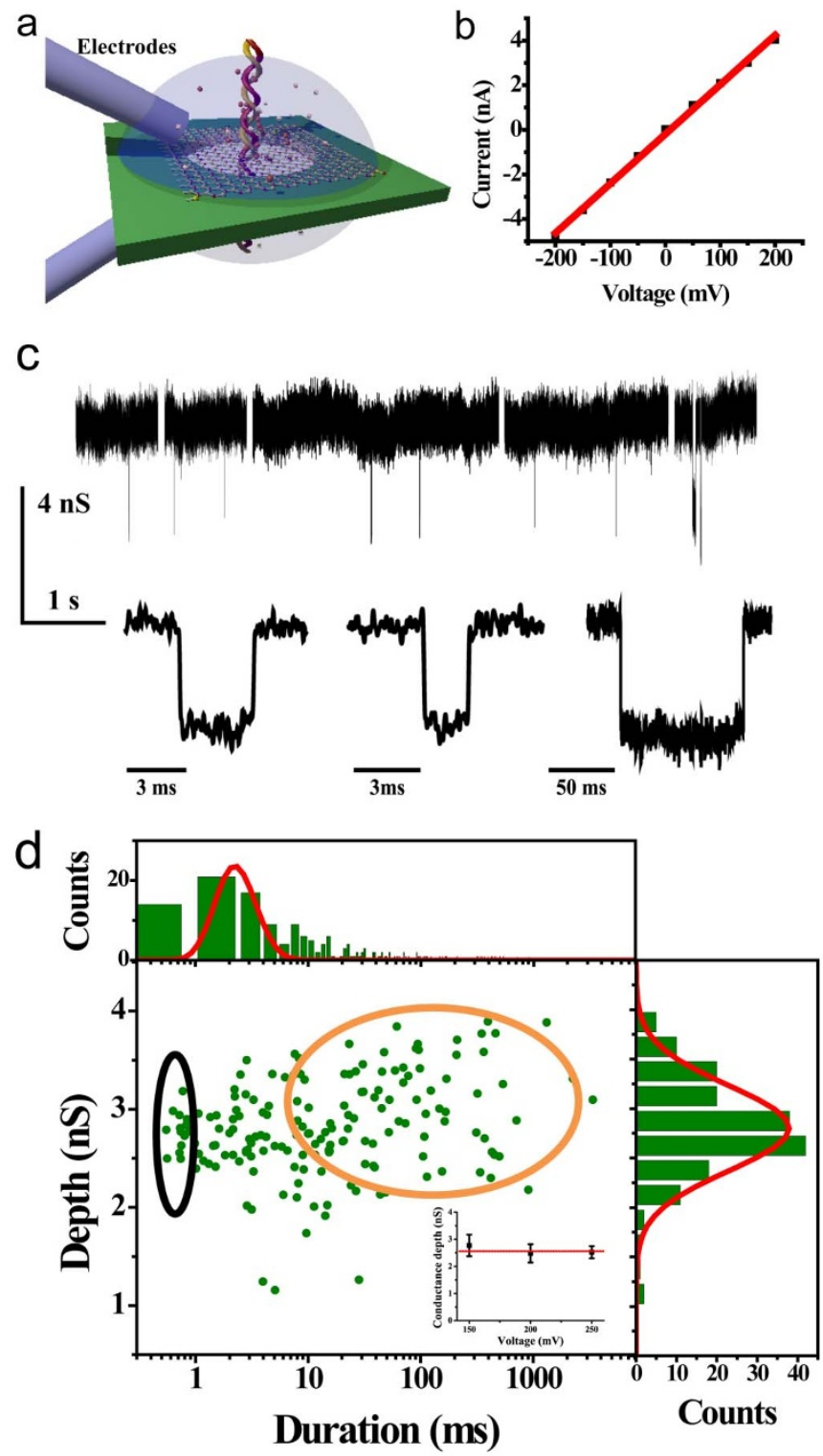

Figure $4 \mid$ (a) Illustration of an h-BN nanopore device. (b) I-V curve measured in a $4 \mathrm{~nm}$ h-BN nanopore with a linear fit. (c) Current traces of DNA translocation in h-BN nanopore and zoom-in plots of typical translocation events. (d) Scatter plot of conductance blockade versus event duration, under a driving voltage of $150 \mathrm{mV}$. The histogram of conductance blockade is shown on the right side, fitted by a Gaussian peak. The histogram of event duration is shown on the top side, fitted by probability density function. The inset of $(\mathrm{d})$ shows conductance blockade of unfolded events as a function of applied driving voltage. 

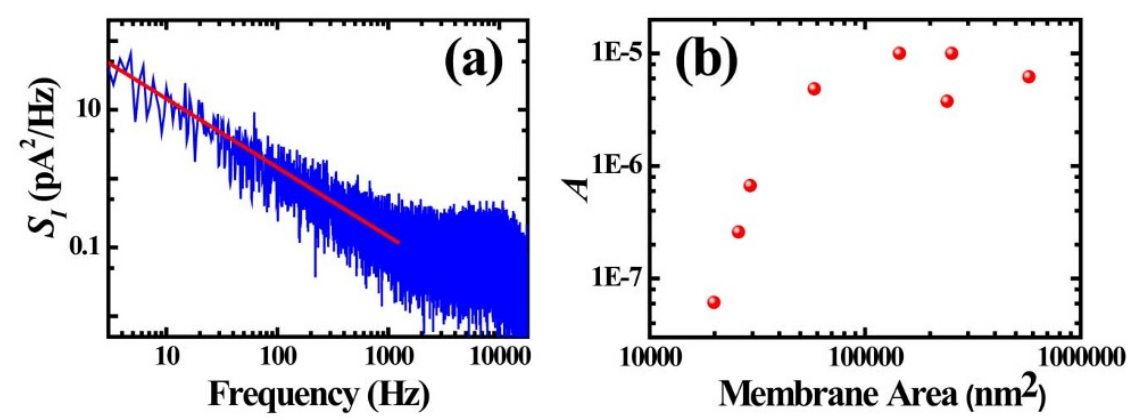

Figure $5 \mid$ (a) Noise spectra of a $10 \mathrm{~nm}$ h-BN nanopore, taken from the current trace which contains DNA translocation events. The current baseline is around $15 \mathrm{nA}$, and the spectra is fitted by Hooge's law. (b) The value of fitted dimensionless parameter $A$ as a function of suspended h-BN membrane area.

$2.8 \mathrm{nS}$, corresponding to the averaged conductance blockade for unfolded DNA molecules. No translocation event of folded DNA was observed in this device since the pore size is too small for the folded DNA molecules to pass through. The averaged conductance blockade is invariant as the driving voltage changes from $150 \mathrm{mV}$ to $250 \mathrm{mV}$, as shown in the inset of Fig. 4(d). On the upper side of the scatter plot is the histogram of translocation time $\Delta \mathrm{t}$. To investigate the electrophoresis parameters of DNA translocation through the h$\mathrm{BN}$ nanopore, we fit the events with density functions of the $1 \mathrm{D}$ based diffusion model ${ }^{26,27}$

$$
P(t)=N \frac{L * e^{-\frac{(L-t v)^{2}}{4 t D}}}{t \sqrt{4 \pi t D}}
$$

Where $N$ is the normalized coefficient of the peak, $v$ is the mean velocity of the molecules, and $D$ is the diffusion constant. The passage length $L=L_{D N A}+H_{\text {eff }}$ is simplified to the DNA length $L_{D N A}(16 \mu \mathrm{m}$ for unfolded $\lambda$-DNA) because of the ultra-small thickness of the h$\mathrm{BN}$ membrane. The fitting result is shown as solid red curve in the plot. The mean translocation speed for DNA translocation is $5.5 \mu \mathrm{m} /$ $\mathrm{ms}$, similar to that in previous report on $\lambda$-DNA translocation through graphene nanopore ${ }^{10}$. The diffusion constant $D$ is fitted to be $8 \mu \mathrm{m}^{2} / \mathrm{ms}$, which is significantly larger than the bulk value of $\lambda$ DNA.

\section{Discussion}

The histogram data do not match the fitting curve perfectly. Translocation events with duration time less than $1 \mathrm{~ms}$ (as contained in the black circle) may be contributed by DNA fragments, while those events with much longer duration time (contained in the orange circle) are due to DNA-nanopore interaction. For quantitative analysis, we discriminate elongated DNA translocation events as those events longer than the peak duration time by $1.5 \sigma$, where $\sigma$ is the full width at the half maximum (FWHM) of the fitted curve. The translocation time of elongated events in Fig. 4(d) is more than $6 \mathrm{~ms}$. The percentage of elongated events adds up to $65 \%$ of all data points in the figure. The average translocation time of the elongated events is $108 \mathrm{~ms}$, about 40 times longer than that of normal events. The anomalously long translocation time, as well as the high percentage, of elongated events indicates a pretty strong interaction between DNA molecules and the h-BN nanopore.

To further investigate such interaction in h-BN nanopores, we examined DNA translocation behavior in pores of different sizes. A moderate $10 \mathrm{~nm}$ (with $\lambda$-DNA) pore and a big $30 \mathrm{~nm}$ (with $10 \mathrm{kbp}$ DNA) pore are fabricated for the test. Both folded and unfolded translocation events are observed in both pores (Figure S4). By analyzing duration time of all unfolded events (Figure S5), the percentage of translocation events undergoes molecule-pore interaction is about $71 \%$ in the $10 \mathrm{~nm}$ pore and about $20 \%$ in the $30 \mathrm{~nm}$ pore. The elongated translocation time is $16 \mathrm{~ms}$ ( 5 times of normal events) for the $10 \mathrm{~nm}$ pore and $0.85 \mathrm{~ms}$ ( 3 times of normal events) for the $30 \mathrm{~nm}$ pore.

Figure 5(a) shows the noise spectrum of a typical h-BN nanopore on a $200 \mathrm{~nm} \mathrm{Si}_{3} \mathrm{~N}_{4}$ window, derived by FFT of real-time current traces. The low frequency noise can be fitted by Hooge's law: $S_{I}=$ $A^{*} I^{2} / f$, where $S_{I}$ is the power spectral density, $I$ is the ionic current, $f$ is the frequency, and $A$ is a dimensionless parameter that can be used to characterize the $1 / f$ noise level. The value of $A$ is derived to be $6.7 \times$ $10^{-7}$ for the $200 \mathrm{~nm}$ window. Interestingly, it is found that $1 / f$ noise in h-BN nanopore is closely related to the size of the suspended membrane area. Figure 5(b) plots the value of $A$ as a function of membrane area, which is obtained by subtracting nanopore area from the $\mathrm{Si}_{3} \mathrm{~N}_{4}$ window area. For a smaller $180 \mathrm{~nm}$ window, the value reduces to $2.6 \times 10^{-7}$; and for a bigger $550 \mathrm{~nm}$ window, it increases to $3.75 \times 10^{-6}$. We noticed that the $1 / f$ noise in a graphene nanopore is related to the size of the supporting $\mathrm{Si}_{3} \mathrm{~N}_{4}$ windows as well ${ }^{12}$. Reducing the window size is not only good for optimizing the $1 / f$ noise, but also help increase the yield of h-BN nanopores. In our practice, the h-BN nanopore device is very fragile if the supporting $\mathrm{Si}_{3} \mathrm{~N}_{4}$ window is bigger than $500 \mathrm{~nm}$, while most nanopores were stable when supported by $\mathrm{Si}_{3} \mathrm{~N}_{4}$ window smaller than $200 \mathrm{~nm}$.

In summary, we have demonstrated the nanopore device fabricated on atomic thick h-BN layer. The h-BN nanopores are of good oxidation resistance and are compatible with UVO treatment. The hydrophilicity of h-BN nanopores is improved by UVO treatment with a reduced contact angle of $26^{\circ}$. Abundant DNA translocation events have been readily detected in such nanopore devices with various pore sizes. Strong DNA-nanopore interaction has been revealed in small nanopores, which slows down the DNA translocation significantly. Smaller supporting windows are desired for h-BN nanopores to reduce the $1 / f$ noise and to improve device stability. The demonstrated performance in such hydrophilic ultra-thin h-BN nanopores suggests further promise towards DNA base discrimination based on ionic current analysis.

\section{Methods}

Growth of h-BN. Hexagonal boron nitride was grown on a copper foil ( $25 \mu \mathrm{m}$ thick, 99.99\%, from Alfa Aesar). After cleaned by acetone, ethanol, isopropanol, and deionized water, the copper foil was placed into a quartz tube at $1050^{\circ} \mathrm{C}$, where borane ammonia (BH3-NH3, from Sigma Aldrich) was carried by mixed gas of $\mathrm{Ar}$ and $\mathrm{H}_{2}$ $(200 / 200 \mathrm{sccm})$ under atmospheric pressure. Hexagonal BN layer formed on the surface of copper foil after $30 \mathrm{~min}$ of growth.

Transfer of h-BN. PMMA solution (PMMA 495, concentration 5\%) was spin-coated on one side of h-BN/copper foils at $3000 \mathrm{rpm}$ for $60 \mathrm{~s}$ and cured at $145^{\circ} \mathrm{C}$ for 5 minutes. The $3 \times 3 \mathrm{~mm}^{2} \mathrm{Cu}$ substrate was then etched away by iron chloride $(1 \mathrm{~mol} / \mathrm{L})$ over a period of 12 hours, leave h-BN/PMMA flakes floating in solution. The flakes were then picked up, washed with deionized water several times, and then transferred onto the target substrates. After transfer, PMMA was dissolved with acetone and the substrate with h-BN layer was rinsed by isopropyl alcohol and dried with nitrogen gas flow.

Nanopore fabrication and characterization. The nanopores were drilled by TEM (JEM 2010F, operated at $200 \mathrm{kV}$ ), focusing on h-BN membranes for about 1-3 s 
under about $500 \mathrm{pA} / \mathrm{cm}^{2}$ current density and a magnification of $800 \mathrm{k}$. After drilling, the electron beam was defocused to take images as quickly as possible. The EELS were taken subsequently with a magnification of $100 \mathrm{k}$ and a diaphragm of $100 \mathrm{~nm}$, under the energy resolution of $0.3 \mathrm{eV}$ and integration time of $1.0 \mathrm{~s}$. For each spectrum, the object astigmatism and zero-peak drifting were carefully calibrated. The pore size can be elaborately controlled. To measure the Raman spectra, the h-BN membrane was transferred onto a silicon wafer in order to avoid strong luminescence from $\mathrm{Si}_{3} \mathrm{~N}_{4}$ substrate. All Raman spectra were taken under $532 \mathrm{~nm}$ laser excitation, with focal size of $2 \mu \mathrm{m}$ and power of $1 \mathrm{~mW}$ (HORIBA, JY6400).

Nanopore experiments. The flow cell, PDMS gaskets and nanopore chips were firstly treated with UVO on both the front and the back side for 15 minutes each and then assembled as soon as possible. KCl-TE buffer (contain $1 \mathrm{M} \mathrm{KCl}, 10 \mathrm{mM}$ Tris- $\mathrm{HCl}$, 1 mM EDTA, $\mathrm{pH}=7.8$ ) was filled into the device immediately. Subsequently, the BN nanopores were characterized by I-V curve, and only those chips providing linear and symmetry $I-V$ curves were selected for DNA translocation experiment. Both double strand $\lambda$-DNA (48.5 kbp) and $10 \mathrm{kbp}$ DNA were diluted to $1 \mathrm{nM}$ by KCl-TE buffer and pre-warmed to $70^{\circ}$ for 1 minute to activate DNA molecules. After injection of DNA buffer, driving voltages between $50 \mathrm{mV}$ and $250 \mathrm{mV}$ were applied across the membranes. The ionic current was detected by an Axopatch amplifier (200B) at acquisition rate of $250 \mathrm{kHz}$, with a $30 \mathrm{kHz} 4$-pole Bessel filter. The recorded translocation events were processed in a Matlab GUI program. The program allows us to view the events one by one and only those events with signal-noise-ratio (SNR) bigger than 8 and have a sharp edge are selected for analysis.

1. Kasianowicz, J. J., Brandin, E., Branton, D. \& Deamer, D. W. Characterization of individual polynucleotide molecules using a membrane channel. Proc. Natl. Acad. Sci. USA 93, 13770-13773 (1996).

2. Cherf, G. M. et al. Automated forward and reverse ratcheting of DNA in a nanopore at 5- $\AA$ precision. Nat. Biotech. 30, 344-348 (2012).

3. Butler, T. Z., Pavlenok, M., Derrington, I. M., Niederweis, M. \& Gundlach, J. H. Single-molecule DNA detection with an engineered MspA protein nanopore. Proc. Natl. Acad. Sci. USA 105, 20647-20652 (2008).

4. Manrao, E. A. et al. Reading DNA at single-nucleotide resolution with a mutant MspA nanopore and phi29 DNA polymerase. Nat. Biotech. 30, 349-353 (2012).

5. Li, J. et al. Ion-beam sculpting at nanometer length scales. Nature 412, 166-169 (2001).

6. Venkatesan, B. M. \& Bashir, R. Nanopore sensors for nucleic acid analysis. Nat. Nanotech. 6, 615-624 (2011)

7. Wanunu, M. Nanopores: A journey towards DNA sequencing. Phy. Life Rev. 9, 125-158 (2012).

8. Gershow, M. \& Golovchenko, J. A. Recapturing and trapping single molecules with a solid-state nanopore. Nat. Nanotech. 2, 775-779 (2007).

9. Garaj, S. et al. Graphene as a subnanometre trans-electrode membrane. Nature 467, 190-193 (2010).

10. Schneider, G. F. et al. DNA translocation through Graphene nanopores. Nano Lett. 10, 3163-3167 (2010)

11. Merchant, C. A. et al. DNA translocation through Graphene nanopores. Nano Lett. 10, 2915-2921 (2010).

12. Garaj, S., Liu, S., Golovchenko, J. A. \& Brantom, D. Molecule-hugging graphene nanopores. Proc. Natl. Acad. Sci. USA 110, 12192-12196 (2013).

13. Schneider, G. F. et al. Tailoring the hydrophobicity of graphene for its use as nanopores for DNA translocation. Nat. Commun. 4, 2619 (2013).

14. Golberg, D. et al. Boron nitride nanotubes and nanosheets. ACS Nano 4 2979-2993 (2010).

15. Chen, Y., Zou, J., Campbell, S. J. \& Caer, G. L. Boron nitride nanotubes: pronounced resistance to oxidation. Appl. Phys. Lett. 84, 2430-2432 (2004).
16. Pakdel, A., Zhi, C., Bando, Y. \& Golberg, D. Low-dimensional boron nitride nanomaterials. Mater. Today 15, 256-265 (2012).

17. Watanabe, K., Taniguchi, T. \& Kanda, H. Direct-bandgap properties and evidence for ultraviolet lasing of hexagonal boron nitride single crystal. Nat. Mater. 3, 404-409 (2004).

18. Lee, C. et al. Frictional characteristics of atomically thin sheets. Science 328, 76-80 (2010).

19. Kim, K. K. et al. Synthesis of monolayer hexagonal boron nitride on $\mathrm{Cu}$ foil using chemical vapor deposition. Nano Lett. 12, 161-166 (2012).

20. Song, L. et al. Large scale growth and characterization of atomic hexagonal boron nitride layers. Nano Lett. 10, 3209-3215 (2010).

21. Nag, A. et al. Graphene analogues of BN: novel synthesis and properties. ACS Nano 4, 1539-1544 (2010).

22. Liu, S. et al. Boron nitride nanopores: highly sensitive DNA single-molecule detectors. Adv. Mater. DOI: 10.1002/adma.201301336 (2013).

23. Gorbachev, R. V. et al. Hunting for monolayer boron nitride: optical and Raman signatures. Small 7, 465-468 (2011).

24. Lin, Q., Painter, O. J. \& Agrawal, G. P. Nonlinear optical phenomena in silicon waveguides: modeling and applications. Opt. Express 15, 16604-16644 (2007).

25. Zhang, E. X. et al. Ozone-exposure and annealing effects on graphene-on- $\mathrm{SiO}_{2}$ transistors. Appl. Phys. Lett. 101, 121601 (2012).

26. Li, J. \& Talaga, D. S. The distribution of DNA translocation times in solid-state nanopores. J. Phys.: Condens. Matter 22, 454129 (2010).

27. Daniel Y. Ling \& Xinsheng Sean Ling. On the distribution of DNA translocation times in solid-state nanopores: an analysis using Schrödinger's first-passage-time theory. J. Phys.: Condens. Matter 25, 375102 (2013).

\section{Acknowledgments}

We are grateful to Laboratory of Microfabrication, Dr. Y. Yao, and Prof. R.C. Yu (Institute of Physics, Chinese Academy of Sciences) for technical assistance. We also thank Dr. Bo Lu, Prof. Qing Zhao, Prof. Dapeng Yu (Peking University, China) for helpful discussion. This work is supported by Hundred Talent Program of Chinese Academy of Sciences, NSFC under Grant No. 61027011, and National Basic Research Program of China under Grant No. 2012 CB933002.

\section{Author contributions}

Z.Z. fabricated nanopores by FIB and TEM, and run the DNA translocation experiments. Y.H. transferred the h-BN layers and built the flow cell setup. H.W. and W.W. prepared the h-BN sample by CVD. Z.X. and X.B. performed TEM experiments and give helpful guide. Z.Z. and X.S. analyzed the data and X.L. designed and initiated the experiments. Z.Z., X.S and X.L. were mainly responsible for the preparing of the manuscript with discussion and comments of other authors.

\section{Additional information}

Supplementary information accompanies this paper at http://www.nature.com/ scientificreports

Competing financial interests: The authors declare no competing financial interests. How to cite this article: Zhou, Z. et al. DNA Translocation through Hydrophilic Nanopore in Hexagonal Boron Nitride. Sci. Rep. 3, 3287; DOI:10.1038/srep03287 (2013).

This work is licensed under a Creative Commons Attribution 3.0 Unported license. To view a copy of this license, visit http://creativecommons.org/licenses/by/3.0 